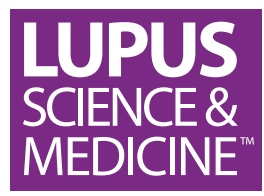

\title{
Impact of systemic lupus erythematosus disease activity, hydroxychloroquine and NSAID on the risk of subsequent organ system damage and death: analysis in a single US medical centre
}

\author{
Deanna D Hill, ${ }^{1}$ Amanda M Eudy, ${ }^{2}$ Peter J Egger, ${ }^{3}$ Qinggong Fu, ${ }^{1}$ \\ Michelle A Petri (i) ${ }^{4}$
}

To cite: Hill DD, Eudy AM, Egger PJ, et al. Impact of systemic lupus erythematosus disease activity, hydroxychloroquine and NSAID on the risk of subsequent organ system damage and death: analysis in a single US medical centre. Lupus Science \& Medicine 2021;8:e000446. doi:10.1136/ lupus-2020-000446

Received 24 September 2020 Revised 26 February 2021 Accepted 11 March 2021

Check for updates

(c) Author(s) (or their employer(s)) 2021. Re-use permitted under CC BY-NC. No commercial re-use. See rights and permissions. Published by BMJ.

${ }^{1}$ Real World Evidence, Epidemiology, GlaxoSmithKline, Collegeville, Pennsylvania, USA ${ }^{2}$ Duke University School of Medicine, Durham, North

Carolina, USA

${ }^{3}$ Real World Evidence, Epidemiology, GlaxoSmithKline, Uxbridge, UK

${ }^{4}$ Department of Medicine, Johns Hopkins University School of Medicine, Baltimore, Maryland, USA

Correspondence to Dr Michelle A Petri; mpetri@ jhmi.edu

\section{ABSTRACT}

Objective To assess the impact of mild-moderate systemic lupus erythematosus (SLE) disease activity during a 12-month period on the risk of death or subsequent organ system damage.

Methods 1168 patients with $\geq 24$ months of follow-up from the Hopkins Lupus Cohort were included. Disease activity in a 12-month observation period was calculated using adjusted mean Safety of Estrogens in Lupus Erythematosus National Assessment (SELENA) version of the SLE Disease Activity Index (SLEDAI), defined as the area under the curve divided by the time interval. Damage accrual in the follow-up period was defined as change in Systemic Lupus International Collaborating Clinics/ American College of Rheumatology Damage Index (SDI) score $\geq 1$ among patients without prior damage. Patients visited the clinic quarterly and had SELENA-SLEDAI and SDI assessed at every visit.

Results During follow-up (median 7 years), $39 \%$ of patients accrued new damage in any organ system (7\% cardiovascular and 3\% renal) and $8 \%$ died. In adjusted models, an increased SELENA-SLEDAl score increased the risk of death $(\mathrm{HR}=1.22,95 \% \mathrm{Cl} 1.13$ to $1.32, \mathrm{p}<0.001)$, renal damage $(\mathrm{HR}=1.24,95 \% \mathrm{Cl} 1.08$ to $1.42, \mathrm{p}=0.003)$ and cardiovascular damage $(\mathrm{HR}=1.17$, $95 \% \mathrm{Cl} 1.07$ to $1.29, \mathrm{p}<0.001)$. Hydroxychloroquine use reduced the risk of death $(\mathrm{HR}=0.46,95 \% \mathrm{Cl} 0.29$ to 0.72 , $\mathrm{p}<0.05)$ and renal damage $(\mathrm{HR}=0.30,95 \% \mathrm{Cl} 0.13$ to $0.68, \mathrm{p}<0.05)$. Non-steroidal anti-inflammatory drug use increased the risk of cardiovascular damage $(\mathrm{HR}=1.66$, $95 \% \mathrm{Cl} 1.04$ to 2.63, $\mathrm{p}<0.05)$. Without prior damage, an increased adjusted mean SELENA-SLEDAI score increased the risk of overall damage accrual $(\mathrm{HR}=1.09,95 \% \mathrm{Cl} 1.04$ to $1.15, p<0.001$ ).

Conclusions Each one-unit increase in adjusted mean SELENA-SLEDAI during a 12-month observation period was associated with an increased risk of death and developing cardiovascular and renal damage.

\section{INTRODUCTION}

Systemic lupus erythematosus (SLE) is an autoimmune disease characterised by a

\section{Key messages}

What is already known about this subject?

- Previous studies have demonstrated that systemic lupus erythematosus (SLE) disease activity and damage accrual are strong predictors of SLE prognosis and survival.

What does this study add?

- This study involved patients with SLE from the large and racially diverse Hopkins Lupus Cohort, composed of $55 \%$ white patients and $39 \%$ patients of Black African Ancestry. This prospective analysis demonstrated that increases in adjusted mean Safety of Estrogens in Lupus Erythematosus National Assessment-SLE Disease Activity Index (SELENASLEDAl) score during a 12-month observational period were associated with an increased risk of death or developing renal or cardiovascular damage.

- Chronic non-steroidal anti-inflammatory drug (NSAID) use resulted in significantly increased risk of developing cardiovascular damage.

- SLE may progress to irreversible damage in selected organ systems among patients with stable, mild-tomoderate SLE disease activity over 7 years.

How might this impact on clinical practice or future developments?

- This analysis demonstrates that, even in patients with mild-to-moderate disease activity, an increase in adjusted mean SELENA-SLEDAI score and NSAID use during a 12-month period increases the risk of death and organ specific damage, highlighting the need for more active measures to manage SLE disease activity over time and to limit NSAID use.

complex and multifactorial aetiology. It affects multiple organ systems, which contributes to the complicated patient burden of SLE. Some patients with SLE experience a waxing and waning pattern of disease activity during the course of disease, whereas others 
have continuous activity. ${ }^{1}$ Cumulative SLE disease activity ${ }^{2}$ and SLE therapies ${ }^{34}$ impact the risk of developing organ system damage and survival. ${ }^{5-7}$ The 5 -year survival among individuals with SLE has increased dramatically in the last four decades to $95 \%,{ }^{7}$ with similar trends observed for 10-year survival. ${ }^{78}$ The impact of irreversible organ system damage in the prognosis of SLE remains a major concern because patients who develop damage are more likely to accrue additional damage and die. ${ }^{9}$ Published research from prospective cohorts has demonstrated that SLE disease activity is a strong predictor of SLE prognosis and survival. ${ }^{1011}$

The objective of this analysis was to investigate whether the pattern of SLE disease activity (as measured by the Safety of Estrogens in Lupus Erythematosus National Assessment (SELENA) version of the SLE Disease Activity Index (SLEDAI)) during a 12-month period after enrolment in a racially diverse cohort of patients prospectively followed quarterly by protocol impacted the risk of developing damage (overall or by specific organ system) and death over time.

\section{METHODS}

\section{Patient population}

Demographic, clinical and laboratory data were collected prospectively in enrolled patients in the Hopkins Lupus Cohort from 1987 to $2010 .{ }^{12-15}$ Cohort patients with a minimum of 24-month follow-up were included in this analysis. Patients were seen by a single rheumatologist in the Hopkins Lupus Center (MAP) and had a clinical diagnosis of SLE according to the revised American College of Rheumatology (ACR) or Systemic Lupus International Collaborating Clinics (SLICC) criteria. ${ }^{16-18}$ The study was approved by the Johns Hopkins University School of Medicine Institutional Review Board. All patients provided informed written consent. Patients with a SLICC/ACR Damage Index (SDI) score $\geq 3$, end-stage kidney disease, history of major organ transplant or malignant neoplasm at cohort entry were excluded from this analysis.

\section{Patient follow-up}

Follow-up time in the cohort was then divided into three discrete periods: (a) background (background period: first 12 months after cohort entry), (b) observation (observation period: second 12 months after cohort entry) and (c) follow-up (follow-up period: remainder of time under observation until damage occurred, death or end of available data). SLE disease duration was defined as the time interval from SLE clinical diagnosis (revised ACR or SLICC criteria) until the end of the follow-up period or death.

\section{Study variables}

Key variables included in the observation period were demographic characteristics, SLE clinical characteristics, laboratory parameters, medication use and comorbid medical conditions (hypertension, diabetes mellitus, obesity, etc.). Hypertension was defined as systolic blood pressure greater than $140 \mathrm{~mm} \mathrm{Hg}$ or diastolic blood pressure greater than 90 $\mathrm{mm} \mathrm{Hg}$ or requiring antihypertensive medication. Diabetes mellitus was defined as requiring an oral hypoglycaemic agent or insulin therapy. Immunosuppressive therapies excluded steroids and included the following: leflunomide, mycophenolate, cyclophosphamide, tacrolimus, azathioprine, methotrexate or rituximab (biologic). Antimalarial therapies were almost exclusively comprised of hydroxychloroquine and non-steroidal anti-inflammatory drug (NSAID) therapy included several drugs (prescription and over-the-counter) in this class, but almost exclusively naproxen. For the aforementioned SLE therapies, use was defined as ever prescribed during the observation period (yes/no). Oral steroid (prednisone) use in the observational period was stratified into the following groups: ever prescribed any dose (yes) or never prescribed (no). Among those prescribed oral steroids, daily dose during the observation period was stratified into two categories: (a) prescribed $>7.5 \mathrm{mg} /$ day at least once during the observation period or (b) only prescribed $\leq 7.5 \mathrm{mg} /$ day during the observation period. Antinuclear antibody positivity (ANA+) was defined as a titre $\geq 1: 80$ and antidouble stranded DNA positivity (antidsDNA+) was defined as a titre $\geq 1: 10$ on the Crithidia luciliae indirect immunofluorescence test.

Disease activity was measured at every clinic visit (quarterly by protocol or more often if clinically warranted) using the SELENA-SLEDAI. ${ }^{19}$ To describe disease activity over time in this analysis, adjusted mean SELENA-SLEDAI ${ }^{20}{ }^{21}$ was calculated from the cumulative area under the curve for each period divided by the time interval under evaluation and reported in months. At least one clinic visit per period was required to estimate adjusted mean SELENA-SLEDAI for each period. Damage accrual was assessed at each visit by the SDI. ${ }^{22}{ }^{23}$

\section{Data analysis}

All statistical analyses were completed in SAS V.9.13 (SAS Institute, Cary, North Carolina, USA).

Continuous variables were reported as median values unless otherwise noted. Categorical variables were described as frequency counts. SLE outcomes of interest included (a) development of overall organ system damage, (b) development of specific organ system damage or (c) death during the follow-up period. The outcome of death was defined as all-cause mortality that may or may not have been clinically attributable to SLE. Damage accrual analyses were restricted to patients without prior damage in the organ system of interest through the end of the observation period. Accrued damage was defined as at least a one $(\geq 1)$ unit increase in SDI score between the observation period and last recorded visit in the follow-up period.

In this analysis, organ specific system damage was restricted to renal, cardiovascular, peripheral vascular, neuropsychiatric, pulmonary, musculoskeletal, seizure or stroke. Damage due to malignancy was not explored as it was an exclusion criterion. Damage accrual in organ systems not commonly associated with death (ocular, 
gastrointestinal, skin, or gonadal failure) was not evaluated in this analysis.

Adjusted Mean SELENA-SLEDAI was our key explanatory variable. Laboratory parameters were not considered in models evaluating the impact of adjusted mean SELENA-SLEDAI on the outcomes of interest since these variables are components of the SELENA-SLEDAI score. Time-varying variables that were measured during the observation period and SLE clinical history variables were individually evaluated in univariate, unadjusted models for the outcomes of death or damage accrual (overall or specific organ systems). When more than one variable was available to evaluate comorbidity status, the variable that was most relevant to the time period of interest was used. If variables were comparable, then both were retained in the univariate analyses and the results from the univariate analysis determined which variable was considered in the multivariable model (when applicable). Variables with a $\mathrm{p}<0.10$ in the univariate analysis were considered as potential covariates in the multivariable models for the outcomes of death or damage accrual. Cox proportional hazard models were used to estimate the impact of adjusted mean SELENA-SLEDAI as a time-dependent variable on the risk of death or developing any new organ damage over time. Kaplan-Meier survival curves were also generated to explore the impact of adjusted mean SELENA-SLEDAI in the observation period on the risk of death over time during the follow-up period.

For each outcome, a multivariable model was created to adjust for well-established potential confounding factors that included age, gender, race, SLE duration, overall SDI score at the start of the follow-up period and ever prescribed oral prednisone $>7.5 \mathrm{mg} /$ day in the observation period. With these factors forced in the model, other covariates identified in the univariate analyses were then evaluated for inclusion in the model using backward elimination methods with the criteria of $\mathrm{p}<0.10$ for entry into the model and $\mathrm{p}<0.05$ for retention. To evaluate racial and gender differences in the association of adjusted mean SELENA-SLEDAI and organ system damage, models were stratified by race and gender (data not shown).

\section{Patient and public involvement}

We did not involve patients and/or the public in this work.

\section{RESULTS}

\section{Characteristics}

Overall, 1168 adult patients with SLE were included in this study. Patients were predominantly women (93\%), $55 \%$ were white and 39\% were of Black African Ancestry (table 1). During the background period, the median (range) adjusted mean SELENA-SLEDAI was $3(0-16)$ and $55 \%$ of patients had mild-to-moderate disease activity, defined by an adjusted mean SELENA-SLEDAI $<3$ (table 1). Median (range) adjusted mean SELENASLEDAI in the observation period was $2(0-13)$ and
Table 1 Demographic and clinical characteristics of the Hopkins Lupus Cohort analytical cohort $(\mathrm{N}=1168)$

\begin{tabular}{lc}
\hline & $\mathbf{N}(\%)$ \\
\hline Women & $1085(92.9)$ \\
Race & \\
$\quad$ White & $643(55.1)$ \\
Black African Ancestry & $460(39.4)$ \\
Other & $65(5.6)$
\end{tabular}

History of seropositive status

\begin{tabular}{|c|c|}
\hline ANA+ & $1075(92.0)$ \\
\hline Anti-dsDNA+ & $591(50.6)$ \\
\hline ANA+ or anti-dsDNA+ & $1093(93.6)$ \\
\hline Current smoker at cohort entry & $202(17.3)$ \\
\hline Past smoker at cohort entry & $465(39.8)$ \\
\hline $\begin{array}{l}\text { Adjusted mean SELENA-SLEDAI }<3 \text { in } \\
\text { background period }\end{array}$ & $641(54.9)$ \\
\hline \multicolumn{2}{|l|}{$\begin{array}{l}\text { SLE therapies* ever prescribed in } \\
\text { observation period }\end{array}$} \\
\hline Oral prednisone (any dose) & $702(60.1)$ \\
\hline Oral prednisone $>7.5 \mathrm{mg} /$ day & $428(36.6)$ \\
\hline $\mathrm{HCQ}$ & $759(65.0)$ \\
\hline NSAID & 447 (38.3) \\
\hline Immunosuppressants $\dagger$ & $262(22.4)$ \\
\hline $\begin{array}{l}\text { Obesity in observation period (BMI } \\
>27.8 \text { for men and } \mathrm{BMI}>27.3 \text { for } \\
\text { women) }\end{array}$ & $604(51.7)$ \\
\hline
\end{tabular}

Hypertension in observation period

$652(55.8)$

(SBP $\geq 140$ or DBP $\geq 90 \mathrm{~mm} \mathrm{Hg}$ )

\begin{tabular}{|c|c|}
\hline \multirow{2}{*}{$\begin{array}{l}\text { Diabetes therapy with oral } \\
\text { hypoglycaemic agent or insulin }\end{array}$} & $118(10.1)$ \\
\hline & Median (range) \\
\hline Age at cohort entry $\ddagger$, years & $36(11-77)$ \\
\hline Age at SLE diagnosis§, years & $31(5-75)$ \\
\hline $\begin{array}{l}\text { Age at last assessment in follow-up } \\
\text { period, years }\end{array}$ & $46(20-85)$ \\
\hline $\begin{array}{l}\text { Adjusted mean SELENA-SLEDAI in } \\
\text { background period }\end{array}$ & $3(0-16)$ \\
\hline $\begin{array}{l}\text { Disease duration from SLE diagnosis to } \\
\text { cohort entry, years }\end{array}$ & $2(0-39)$ \\
\hline $\begin{array}{l}\text { Disease duration from SLE diagnosis to } \\
\text { last assessment, years }\end{array}$ & $11(2-48)$ \\
\hline $\begin{array}{l}\text { Follow-up time from cohort entry to last } \\
\text { assessment, years }\end{array}$ & $7(2-23)$ \\
\hline
\end{tabular}

${ }^{*}$ Categories not mutually exclusive.

†Leflunomide, mycophenolate, cyclophosphamide, tacrolimus, azathioprine, methotrexate or rituximab (biologic).

fLess than $2 \%$ of cohort enrolled $<18$ years of age.

$\S$ Less than $5 \%$ of cohort diagnosed with SLE $<18$ years of age.

ANA, antinuclear antibodies; BMI, body mass index in $\mathrm{kg} / \mathrm{m}^{2}$; DBP, diastolic blood pressure; dsDNA, double-stranded DNA; $\mathrm{HCQ}$, hydroxychloroquine; NSAID, non-steroidal anti-inflammatory drug; SBP, systolic blood pressure; SELENA-SLEDAI, Safety of Estrogens in Lupus Erythematosus National Assessment-SLE Disease Activity Index; SLE, systemic lupus erythematosus. 


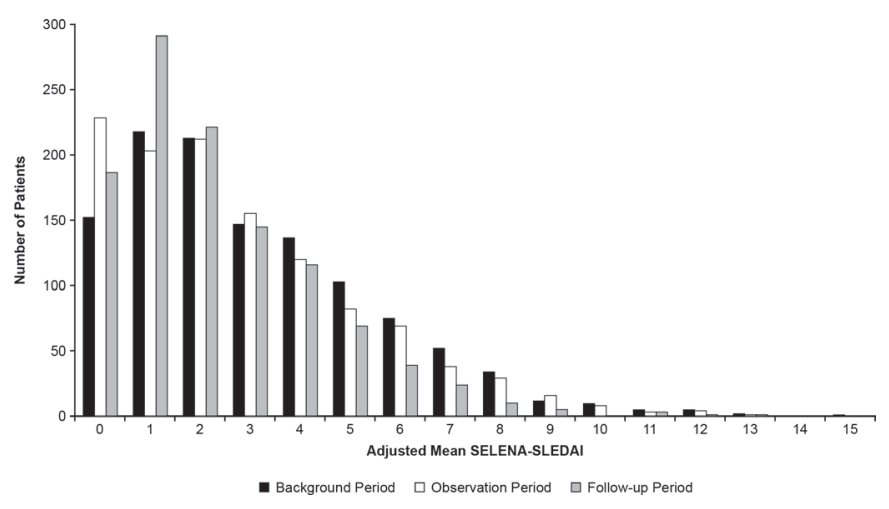

Figure 1 Distribution of adjusted mean SELENA-SLEDAI stratified by period $(\mathrm{N}=1168)$. SELENA-SLEDAI, Safety of Estrogens in Lupus Erythematosus National AssessmentSystemic Lupus Erythematosus Disease Activity Index.

remained the same during the follow-up period. The distribution of adjusted mean SELENA-SLEDAI, stratified by period, is shown in figure 1 .

\section{Death}

Ninety-two of 1168 patients (8\%) died during the follow-up period (table 2). When exploring the association between adjusted mean SELENA-SLEDAI in the observation period and risk of death in the follow-up period, an adjusted mean SELENA-SLEDAI $\geq 5$ significantly increased the risk of death after 7 years of follow-up (Log rank $\mathrm{p}<0.001$; figure 2 ). In the multivariable model, each one-unit adjusted mean SELENA-SLEDAI increase in the observation period was significantly associated with a $22 \%$ increased risk of death in the follow-up period $(\mathrm{HR}=1.22,95 \%$ CI 1.13 to $1.32, \mathrm{p}<0.001$; table 2$)$. Use of hydroxychloroquine in the observation period was significantly associated with a $54 \%$ lower risk of death in the follow-up period ( $\mathrm{HR}=0.46,95 \%$ CI 0.29 to $0.72, \mathrm{p}<0.05$; table 2 ). The following variables were considered but not retained in the model: have a history of smoking at cohort entry, hypertension and diabetes.

\section{Overall damage accrual}

After restricting the analysis (univariate and multivariable) to patients without a prior history of overall damage ( $\mathrm{SDI}=0 ; \mathrm{n}=888$ ) at the start of the follow-up period, more than one-third $(39 \%)$ of patients developed damage $(\mathrm{SDI} \geq 1)$ in any organ system by the end of the follow-up period (table 2). In multivariable models restricted to patients without prior damage in the organ system at the start of the follow-up period, adjusted for the effects of age, race, gender, SLE duration and ever prescribed $>7.5 \mathrm{mg} /$ day oral prednisone during the observation period, a one-unit increase in adjusted mean SELENASLEDAI during the observation period was significantly associated with an increased risk of accruing subsequent overall damage $(\mathrm{HR}=1.09,95 \%$ CI 1.04 to $1.15, \mathrm{p}<0.001$; table 2). Among women without prior damage at the start of the follow-up period, adjusted mean SELENA-SLEDAI during the observation period was associated with an increased risk of accruing subsequent overall damage
( $\mathrm{HR}=1.08,95 \%$ CI 1.03 to $1.13, \mathrm{p}=0.002$; data not shown $)$. No significant differences were observed for the outcomes of interest when stratified by race $(\mathrm{HR}=1.07,95 \%$ CI 0.99 to $1.16, \mathrm{p}=0.089$ in white patients and $\mathrm{HR}=1.04,95 \% \mathrm{CI}$ 0.96 to $1.12, p=0.343$ in patients of Black African Ancestry; data not shown).

\section{Renal damage accrual}

Approximately 3\% of patients without renal damage in the observational period developed renal damage $(\mathrm{SDI} \geq 1 ; \mathrm{n}=1147)$ in the follow-up period (table 2$)$. Each one-unit increase in adjusted mean SELENA-SLEDAI during the observation period significantly increased the risk of renal damage by $24 \%(\mathrm{HR}=1.24,95 \%$ CI 1.08 to 1.42, $\mathrm{p}=0.003$; table 2 ) in the follow-up period. Ever use of hydroxychloroquine in the observation period was significantly associated with lowering the risk of renal damage in the follow-up period by $70 \%$ ( $\mathrm{HR}=0.30,95 \%$ CI 0.13 to $0.68, \mathrm{p}<0.05$; table 2$)$. When we restricted the analysis to women, the results did not change $(\mathrm{HR}=1.23,95 \%$ CI 1.06 to $1.42, \mathrm{p}=0.005$ ). It was not possible to evaluate renal damage among men due to only one man having developed renal damage.

\section{Cardiovascular damage accrual}

Subsequent cardiovascular damage $(\mathrm{SDI} \geq 1 ; \mathrm{n}=1135)$ was observed in approximately $7 \%$ of patients during the follow-up period (table 2). A one-unit increase in adjusted mean SELENA-SLEDAI during the observation period was associated with a $17 \%(\mathrm{HR}=1.17,95 \%$ CI 1.07 to 1.29 , $\mathrm{p}<0.001$; table 2) significant increased risk of cardiovascular damage accrual in the adjusted model. Patients who had been prescribed NSAID therapies in the observation period had a $66 \%(\mathrm{HR}=1.66,95 \%$ CI 1.04 to 2.63 ; table 2) increased risk of cardiovascular damage accrual. On further exploration, patients with any antihypertensive use in the observation period had an $81 \%(\mathrm{HR}=1.81$, 95\% CI 1.09 to 3.02, $\mathrm{p}<0.05)$ significant increased risk of cardiovascular damage accrual.

\section{Other organ system or subsystem damage accrual}

Adjusted mean SELENA-SLEDAI during the observation period did not impact the risk of developing damage in the following organ systems or subsystems in our analysis: peripheral vascular $(\mathrm{HR}=1.12,95 \%$ CI 0.96 to 1.32 , $\mathrm{p}=0.154)$, pulmonary $(\mathrm{HR}=0.96,95 \%$ CI 0.87 to 1.07 , $\mathrm{p}=0.484)$, neuropsychiatric $(\mathrm{HR}=1.01,95 \%$ CI 0.92 to 1.10, $\mathrm{p}=0.880)$, musculoskeletal $(\mathrm{HR}=1.05$, 95\% CI 0.98 to $1.12, \mathrm{p}=0.194)$, stroke or seizure (data not shown).

\section{DISCUSSION}

This analysis described the impact of disease activity during a 12-month period on the subsequent risk of death or organ system damage in a large, prospective cohort of racially diverse patients with SLE. This study included a large proportion of patients of Black African Ancestry (39.4\%), which contrasts with previous Spanish studies (eg, RELESSER cohort) ${ }^{24-26}$ where the majority of 
Table 2 Final models for adjusted mean SELENA-SLEDAI in the observation period as predictors of death/organ system damage in the follow-up period

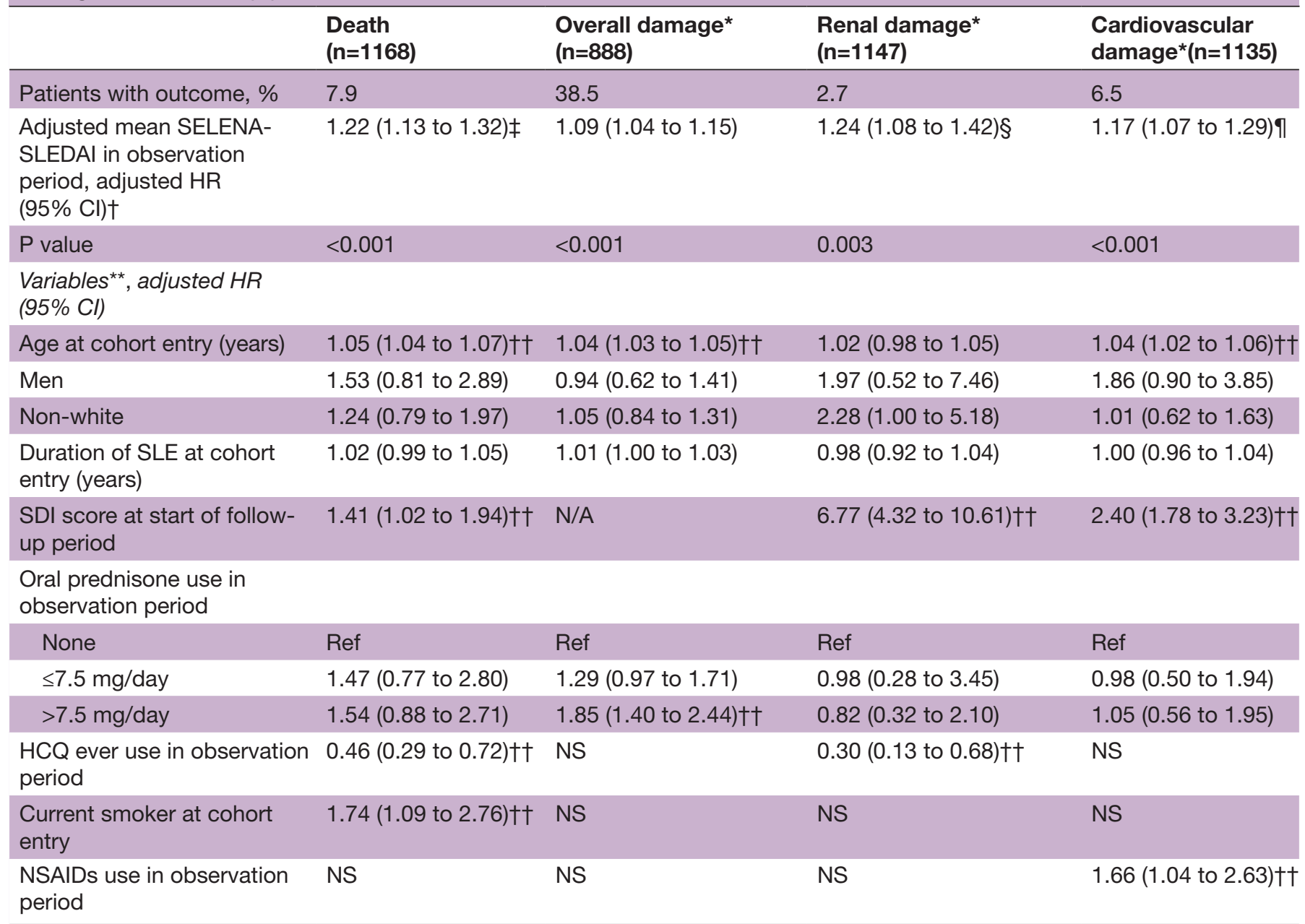

*In patients without a prior history of damage in the organ system of interest at the start of the follow-up period.

THR for a one-unit increase in adjusted mean SELENA-SLEDAl during the observation periods. Models are adjusted for age at cohort entry (years), gender, non-white, duration of SLE at cohort entry (years), damage accrued (SDI score) in any other organ system but the outcome of interest through the end of the observation period (excluding the outcome of overall damage in any organ system), oral prednisone use in observation period (>7.5 mg/day).

$\ddagger$ Additional model adjustments include ever use of hydroxychloroquine during the observation period and current smoker at cohort entry. §Additional model adjustments include ever use of hydroxychloroquine during the observation period.

ๆAdditional model adjustments include NSAID use during the observation period.

${ }^{*}$ SLE therapies defined as ever use during observation period.

††P $<0.05$.

$\mathrm{Cl}$, confidence interval; $\mathrm{HCQ}$, hydroxychloroquine; HR, Cox proportional hazards ratio; NS, not a statistically significant predictor in the final Cox model; NSAID, non-steroidal anti-inflammatory drug; Ref, reference group; SDI, SLICC/ACR damage index; SELENA-SLEDAI, Safety of Estrogens in Lupus Erythematosus National Assessment-SLE Disease Activity Index; SLE, systemic lupus erythematosus.

patients were white $(>90 \%)$, and Latin American studies (eg, GLADEL), ${ }^{27-29}$ which had lower percentages of African-Latin American patients $(<13 \%)$. In this cohort of patients with SLE and mild-to-moderate disease activity at cohort entry, adjusted mean SELENA-SLEDAI measured during a prior 12-month period (corresponding to the length of a typical Phase III trial) significantly impacted the risk of renal and cardiovascular damage accrual (SDI $\geq 1$ ) and risk of death in the follow-up period. In previous studies, patients of Black African Ancestry were reported to experience a higher prevalence of SLE, greater disease severity, higher risk of cardiovascular events, greater organ damage and higher mortality rates, compared with white patients. ${ }^{30-37}$ In the current study, although non-white (Black African Ancestry and other race) patients had higher disease activity in the follow-up period compared with white patients, no significant racial differences were observed for the outcomes of interest. Therefore, results from this cohort provide an important contribution in characterising non-white patient populations. In adjusted Cox regression models, a one-unit increase in adjusted mean SELENA-SLEDAI during the 12-month observation period was associated with a $22 \%$ increased risk of death and a $17 \%$ and $24 \%$ increased 


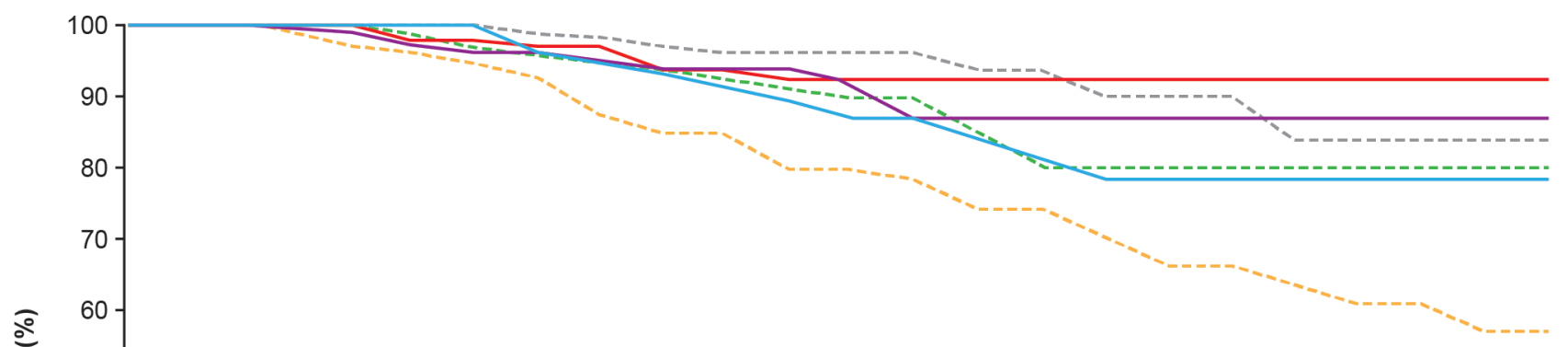

Log rank $p$-value $<0.001$

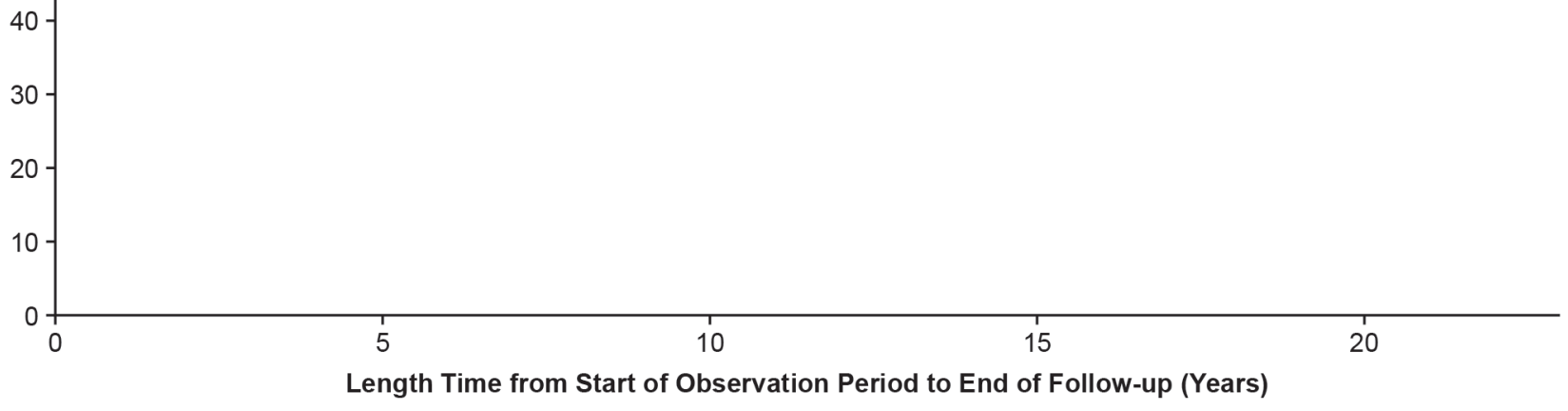

-- AMS $<1$ - AMS 1 -- AMS 2 - AMS 3 - AMS 4 --. AMS 5+

Figure 2 Kaplan-Meier curve: time to death by adjusted mean SELENA-SLEDAI score (N=1168). AMS, adjusted mean SELENA-SLEDAI; SELENA-SLEDAI, Safety of Estrogens in Lupus Erythematosus National Assessment-Systemic Lupus Erythematosus Disease Activity Index.

risk of subsequent cardiovascular and renal damage accrual, respectively. Adjusted mean SELENA-SLEDAI in the observation period was associated with overall damage accrual after adjustment for age, gender, race, SLE duration, ever prescribed oral prednisone therapy $>7.5 \mathrm{mg}$ /day in observation period and SDI at the start of the follow-up period. Hydroxychloroquine use in the observation period was associated with a $54 \%$ and $70 \%$ decreased risk of death and renal damage, respectively, and NSAID use in the observation period increased the risk for cardiovascular damage accrual by approximately $70 \%$.

Adjusted mean SELENA-SLEDAI in a 12-month prior period increased the risk of developing new renal and cardiovascular damage accrual ( $\mathrm{SDI} \geq 1)$ and risk of death, which corroborates findings from another published Hopkins Lupus Cohort analysis with different analytical methods. ${ }^{38}$ Other studies have demonstrated that high disease activity increased the risk of poor SLE outcomes including accrual of overall organ damage $\mathrm{e}^{2039-44}$ and risk of death. ${ }^{704245}$ The findings in this analysis corroborate the influence of disease activity for renal and cardiovascular damage accrual and death and also extend the findings to patients with SLE and mild-to-moderate disease activity.

Although $61.5 \%$ of the cohort remained free of any organ system damage through the end of the follow-up period, a reasonable proportion of patients with SLE with mild-to-moderate disease activity at cohort entry and through the start of the follow-up period accrued damage (renal and cardiovascular) $(9.2 \%)$ in a relatively short time (median 7 years). Similarly, $8 \%$ of patients died during follow-up despite overall mild-to-moderate disease activity in the 24 months after cohort entry.

Detailed methods from real-world, large, prospective SLE cohorts have been described elsewhere ${ }^{10-134647}$ and have contributed to SLE disease understanding over the past four decades. It is well recognised that damage accrual, due to active inflammation, comorbidities, previous SLE disease activity and/or exposure to SLE therapies (particularly corticosteroids), is an important prognostic factor for death and has a clear impact on burden of disease for patients, ${ }^{1520404849}$ but the time for interval for damage to manifest has varied somewhat between different studies. Rahman et al found that patients with SLE exhibiting damage within the first year of admission to the clinic had a higher mortality rate after 10 years as compared with patients with no early damage, ${ }^{50}$ whereas results from Gladman et al suggested that damage accrued gradually over the 15 years of follow-up. ${ }^{2}$ In a study by Becker-Merok and Nossent, damage accrual occurred in $54 \%$ of patients with SLE in a linear fashion over the first 10 years of the disease. ${ }^{42}$ Similarly, a Swedish study also observed that $54 \%$ of patients with SLE had damage accrual in the first 5 years after diagnosis. ${ }^{51}$ Our findings demonstrate that SLE may progress to accrual of irreversible damage in selected organ systems among patients with stable, mild-to-moderate disease activity over 7 years. Our study confirms the results from a Norwegian SLE cohort that showed disease activity at baseline was a predictor of accrued organ damage that occurred over 2 years of follow-up in a small cohort of patients with 
relatively stable disease activity ${ }^{52}$ but extends them to specific organ damage subtypes. Unlike the findings from the SLICC inception cohort, we did not observe a pattern of decreasing disease activity over the first 5 years in conjunction with organ damage accrual. ${ }^{10}$ This may be in part due to the combination of inception and prevalent patients with SLE at the start of the observation period in this Hopkins Lupus Cohort analysis.

Subsequent accrual of organ system damage attributed to active SLE disease has been observed in several SLE prospective cohorts of patients with SLE receiving care from rheumatology specialty centres. ${ }^{510} 11204853-55$ One strength of this analysis is that Hopkins Lupus Cohort patients had clinic visits on average every 3 months per registry protocol. Ibañez et al recently demonstrated that adjusted mean SELENA-SLEDAI derived from quarterly clinic visits was more reliable than adjusted mean SELENA-SLEDAI based on more infrequent clinic visits. ${ }^{56}$

We recognise the limitations of this analysis and similar evaluations in prospective SLE cohorts. One limitation is that all patients in this analysis received care at a single tertiary medical centre, under the care of a single provider, and their SLE clinical characteristics, and hence our findings, may not extend to all patients with SLE. Furthermore, although we attempted to adjust for the effects of known risk factors and potential confounders, unmeasured or residual confounding factors may have influenced our findings.

We observed that adjusted mean SELENA-SLEDAI during a prior 12-month period impacted the risk of death and developing damage in a racially diverse cohort of patients with SLE, who had on average mildto-moderate disease activity during a median follow-up of 7 years, after adjusting for potential confounders. Exposure to hydroxychloroquine during a prior 12-month period decreased the risk of death and developing renal damage. The London University College Hospital Lupus Cohort reported that more than half of patients with SLE were prescribed hydroxychloroquine during a 12-month observation period and this exposure was also associated with a decreased risk of death (50\% reduction) and renal damage accrual (47\% reduction) in adjusted models. ${ }^{11} \mathrm{~A}$ new finding in our analysis was the $66 \%$ increased risk of cardiovascular organ damage accrual associated with NSAID use in the previous 12 months, after controlling for the effects of other covariates in the model. It has been reported that NSAIDs may negatively affect the cardiovascular system, yet they also decrease inflammation, which is an independent risk factor in cardiovascular pathology. ${ }^{57}$ In our analysis, we observed that chronic NSAID use, commonly taken by patients with SLE to alleviate musculoskeletal pain, resulted in a significantly increased risk of cardiovascular damage accrual. NSAID use is also correlated with an increase in blood pressure, which may have an effect on cardiovascular damage. ${ }^{58}$ Indeed, in our study, we observed a significant increase in cardiovascular damage accrual with antihypertensive use. This may suggest that the known cardiovascular risk of NSAIDs in the general population is also applicable to patients with SLE ${ }^{59-62}$ and highlights the importance of assessing cardiovascular risk in this patient population.

In summary, our findings corroborate other published data that demonstrated that cumulative SLE disease activity over time impacted risk of developing organ damage (ie, SLE prognosis) and was associated with an increased risk of death. Furthermore, the findings suggest routine clinical care and SLE disease management, characterised by minimal fluctuations in disease activity, may not prevent development of organ damage or risk of premature death. An increase in adjusted mean SELENASLEDAI score during a 12-month period increased the risk of death and developing renal and cardiovascular organ system damage, even in patients with mild-tomoderate disease severity, which underscores the need for active measures to manage SLE disease activity over time.

Acknowledgements Medical writing support was provided by Helen Taylor, of Fishawack Indicia Ltd, UK, part of Fishawack Health, and was funded by GSK.

Contributors Conception or design: DDH, PJE, QF, MAP and AME. Acquisition of data: MAP. Data analysis or interpretation: DDH, PJE, QF, MAP and AME.

Funding The Hopkins Lupus Cohort is supported by grants R01AR043727 and R01AR069572 from the National Institutes of Health (NIH). This work was funded by GSK Study Number: WEUKBRE4566. Johns Hopkins University School of Medicine received a research grant to support this study. AME is currently supported by $\mathrm{NIH}$ NCATS Award Number 1KL2TR002554.

Competing interests DDH, PJE and QF were paid employees of GlaxoSmithKline (GSK) with stock options and conducted the study as part of their employment using GSK resources. AME was a paid contractor for GSK. MAP was a paid consultant to GSK.

Patient and public involvement Patients and/or the public were not involved in the design, or conduct, or reporting, or dissemination plans of this research. Patient consent for publication Not required.

Ethics approval The study was approved by the Johns Hopkins University School of Medicine Institutional Review Board (Study number NA_00039294).

Provenance and peer review Not commissioned; externally peer reviewed. Data availability statement Data are available on reasonable request.

Author note DDH current affiliation is Center for Observational and Real-World Evidence, Pharmacoepidemiology, Merck \& Co, Inc, Kenilworth, New Jersey, USA.

Open access This is an open access article distributed in accordance with the Creative Commons Attribution Non Commercial (CC BY-NC 4.0) license, which permits others to distribute, remix, adapt, build upon this work non-commercially, and license their derivative works on different terms, provided the original work is properly cited, appropriate credit is given, any changes made indicated, and the use is non-commercial. See: http://creativecommons.org/licenses/by-nc/4.0/.

ORCID iD

Michelle A Petri http://orcid.org/0000-0003-1441-5373

\section{REFERENCES}

1 Barr SG, Zonana-Nacach A, Magder LS, et al. Patterns of disease activity in systemic lupus erythematosus. Arthritis Rheum 1999;42:2682-8.

2 Gladman DD, Urowitz MB, Rahman P, et al. Accrual of organ damage over time in patients with systemic lupus erythematosus. $J$ Rheumatol 2003;30:1955-9.

3 Thamer M, Hernán MA, Zhang Y, et al. Prednisone, lupus activity, and permanent organ damage. J Rheumatol 2009;36:560-4.

4 Zonana-Nacach A, Barr SG, Magder LS, et al. Damage in systemic lupus erythematosus and its association with corticosteroids. Arthritis Rheum 2000;43:1801-8. 
5 Nossent J, Cikes N, Kiss E, et al. Current causes of death in systemic lupus erythematosus in Europe, 2000--2004: relation to disease activity and damage accrual. Lupus 2007;16:309-17.

6 Urowitz MB, Gladman DD, Tom BDM, et al. Changing patterns in mortality and disease outcomes for patients with systemic lupus erythematosus. J Rheumatol 2008;35:2152-8.

7 Kasitanon N, Magder LS, Petri M. Predictors of survival in systemic lupus erythematosus. Medicine 2006;85:147-56.

8 Cervera R, Khamashta MA, Font J, et al. Morbidity and mortality in systemic lupus erythematosus during a 10-year period: a comparison of early and late manifestations in a cohort of 1,000 patients. Medicine 2003;82:299-308.

9 Alarcón GS, Roseman JM, McGwin G, et al. Systemic lupus erythematosus in three ethnic groups. XX. damage as a predictor of further damage. Rheumatology 2004;43:202-5.

10 Urowitz MB, Gladman DD, Ibañez D, et al. Evolution of disease burden over five years in a multicenter inception systemic lupus erythematosus cohort. Arthritis Care Res 2012;64:132-7.

11 Lopez R, Davidson JE, Beeby MD, et al. Lupus disease activity and the risk of subsequent organ damage and mortality in a large lupus cohort. Rheumatology 2012;51:491-8.

12 Petri M. Hopkins lupus cohort. 1999 update. Rheum Dis Clin North Am 2000;26:199-213. v.

13 Petri M. Lupus in Baltimore: evidence-based 'clinical pearls' from the Hopkins Lupus Cohort. Lupus 2005:14:970-3.

14 Petri M. Monitoring systemic lupus erythematosus in standard clinical care. Best Pract Res Clin Rheumatol 2007;21:687-97.

15 Al Sawah S, Zhang X, Zhu B, et al. Effect of corticosteroid use by dose on the risk of developing organ damage over time in systemic lupus erythematosus-the Hopkins lupus cohort. Lupus Sci Med 2015;2:e000066.

16 Hochberg MC. Updating the American College of rheumatology revised criteria for the classification of systemic lupus erythematosus. Arthritis Rheum 1997;40:40:1725.

17 Petri M, Orbai A-M, Alarcón GS, et al. Derivation and validation of the systemic lupus international collaborating clinics classification criteria for systemic lupus erythematosus. Arthritis Rheum 2012;64:2677-86.

18 Tan EM, Cohen AS, Fries JF, et al. The 1982 revised criteria for the classification of systemic lupus erythematosus. Arthritis Rheum 1982;25:1271-7.

19 Petri M, Kim MY, Kalunian KC, et al. Combined oral contraceptives in women with systemic lupus erythematosus. $N$ Engl $\mathrm{J} \mathrm{Med}$ 2005;353:2550-8.

20 Ibañez D, Gladman DD, Urowitz MB. Adjusted mean systemic lupus erythematosus disease activity Index-2K is a predictor of outcome in SLE. J Rheumatol 2005;32:824-7.

21 Ibañez D, Urowitz MB, Gladman DD. Summarizing disease features over time: I. adjusted mean SLEDAI derivation and application to an index of disease activity in lupus. J Rheumatol 2003;30:1977-82.

22 Gladman DD, Urowitz MB. The SLICC/ACR damage index: progress report and experience in the field. Lupus 1999;8:632-7.

23 Gladman D, Ginzler E, Goldsmith C, et al. Systemic lupus international collaborative clinics: development of a damage index in systemic lupus erythematosus. J Rheumatol 1992:19:1820-1.

24 Rúa-Figueroa Î́nigo, Richi P, López-Longo FJ, et al. Comprehensive description of clinical characteristics of a large systemic lupus erythematosus cohort from the Spanish rheumatology Society lupus registry (RELESSER) with emphasis on complete versus incomplete lupus differences. Medicine 2015;94:e267.

25 Pego-Reigosa JM, Lois-Iglesias A, Rúa-Figueroa Íñigo, et al. Relationship between damage clustering and mortality in systemic lupus erythematosus in early and late stages of the disease: cluster analyses in a large cohort from the Spanish Society of rheumatology lupus registry. Rheumatology 2016;55:1243-50.

26 Riveros Frutos A, Holgado S, Sanvisens Bergé A, et al. Late-onset versus early-onset systemic lupus: characteristics and outcome in a national multicentre register (RELESSER). Rheumatology 2020. doi:10.1093/rheumatology/keaa477. [Epub ahead of print: 27 Oct 2020].

27 Pons-Estel BA, Catoggio LJ, Cardiel MH, et al. The GLADEL multinational Latin American prospective inception cohort of 1,214 patients with systemic lupus erythematosus: ethnic and disease heterogeneity among "Hispanics". Medicine 2004;83:1-17.

28 Ugarte-Gil MF, Pons-Estel GJ, Molineros J, et al. Disease features and outcomes in United States lupus patients of Hispanic origin and their Mestizo counterparts in Latin America: a commentary. Rheumatology 2016;55:436-40.

29 Ugarte-Gil MF, Wojdyla D, Pons-Estel GJ, et al. Remission and low disease activity status (LDAS) protect lupus patients from damage occurrence: data from a multiethnic, multinational Latin American lupus cohort (GLADEL). Ann Rheum Dis 2017;76:2071-4.
30 Stojan G, Petri M. Epidemiology of systemic lupus erythematosus: an update. Curr Opin Rheumatol 2018;30:144-50.

31 Barbhaiya M, Feldman CH, Guan H, et al. Race/ethnicity and cardiovascular events among patients with systemic lupus erythematosus. Arthritis Rheumatol 2017;69:1823-31.

32 Lewis MJ, Jawad AS. The effect of ethnicity and genetic ancestry on the epidemiology, clinical features and outcome of systemic lupus erythematosus. Rheumatology 2017;56:i67-77.

33 Munroe ME, Vista ES, Merrill JT, et al. Pathways of impending disease flare in African-American systemic lupus erythematosus patients. J Autoimmun 2017;78:70-8.

34 Rees F, Doherty M, Grainge MJ, et al. The worldwide incidence and prevalence of systemic lupus erythematosus: a systematic review of epidemiological studies. Rheumatology 2017;56:1945-61.

35 Pons-Estel GJ, Alarcón GS, Scofield L, et al. Understanding the epidemiology and progression of systemic lupus erythematosus. Semin Arthritis Rheum 2010;39:257-68.

36 Uribe AG, Alarcón GS. Ethnic disparities in patients with systemic lupus erythematosus. Curr Rheumatol Rep 2003;5:364-9.

37 González LA, Toloza SMA, McGwin G, et al. Ethnicity in systemic lupus erythematosus (SLE): its influence on susceptibility and outcomes. Lupus 2013;22:1214-24.

38 Watson P, Brennan A, Birch $\mathrm{H}$, et al. An integrated extrapolation of long-term outcomes in systemic lupus erythematosus: analysis and simulation of the Hopkins lupus cohort. Rheumatology 2015;54:623-32.

39 Tsang-A-Sjoe MWP, Bultink IEM, Heslinga M, et al. Both prolonged remission and lupus low disease activity state are associated with reduced damage accrual in systemic lupus erythematosus. Rheumatology 2017;56:121-8.

40 Stoll T, Sutcliffe N, Mach J, et al. Analysis of the relationship between disease activity and damage in patients with systemic lupus erythematosus--a 5-yr prospective study. Rheumatology 2004;43:1039-44.

41 Toloza SMA, Roseman JM, Alarcón GS, et al. Systemic lupus erythematosus in a multiethnic US cohort (LUMINA): XXII. predictors of time to the occurrence of initial damage. Arthritis Rheum 2004;50:3177-86.

42 Becker-Merok A, Nossent HC. Damage accumulation in systemic lupus erythematosus and its relation to disease activity and mortality. $J$ Rheumatol 2006:33:1570-7.

43 Peschken CA, Katz SJ, Silverman E, et al. The 1000 Canadian faces of lupus: determinants of disease outcome in a large multiethnic cohort. J Rheumatol 2009;36:1200-8.

44 Taraborelli M, Cavazzana I, Martinazzi N, et al. Organ damage accrual and distribution in systemic lupus erythematosus patients followedup for more than 10 years. Lupus 2017;26:1197-204.

45 Cardoso CRL, Signorelli FV, Papi JAS, et al. Initial and accrued damage as predictors of mortality in Brazilian patients with systemic lupus erythematosus: a cohort study. Lupus 2008;17:1042-8.

46 Alarcón GS. Lessons from LUMINA: a multiethnic US cohort. Lupus 2008:17:971-6.

47 Urowitz MB, Gladman DD. Contributions of observational cohort studies in systemic lupus erythematosus: the University of Toronto lupus clinic experience. Rheum Dis Clin North Am 2005;31:211-21.

48 Alarcón GS, McGwin G, Bartolucci AA, et al. Systemic lupus erythematosus in three ethnic groups. IX. Differences in damage accrual. Arthritis Rheum 2001;44:2797-806.

49 Bruce IN, O'Keeffe AG, Farewell V, et al. Factors associated with damage accrual in patients with systemic lupus erythematosus: results from the systemic lupus international collaborating clinics (SLICC) inception cohort. Ann Rheum Dis 2015;74:1706-13.

50 Rahman P, Gladman DD, Urowitz MB, et al. Early damage as measured by the SLICC/ACR damage index is a predictor of mortality in systemic lupus erythematosus. Lupus 2001;10:93-6.

51 Nived O, Jönsen A, Bengtsson AA, et al. High predictive value of the systemic lupus international collaborating Clinics/American College of rheumatology damage index for survival in systemic lupus erythematosus. J Rheumatol 2002;29:1398-400.

52 Gilboe IM, Kvien TK, Husby G. Disease course in systemic lupus erythematosus: changes in health status, disease activity, and organ damage after 2 years. $J$ Rheumatol 2001;28:266-74.

53 Mimica M, Barra I, Ormeño R, et al. Predictors of damage accrual in systemic lupus erythematosus: a longitudinal observational study with focus on neuropsychological factors and anti-neuronal antibodies. Clin Rheumatol 2019;38:3129-37.

54 Petri M, Purvey S, Fang $\mathrm{H}$, et al. Predictors of organ damage in systemic lupus erythematosus: the Hopkins lupus cohort. Arthritis Rheum 2012;64:4021-8.

55 Segura BT, Bernstein BS, McDonnell T, et al. Damage accrual and mortality over long-term follow-up in 300 patients with systemic 
lupus erythematosus in a multi-ethnic British cohort. Rheumatology 2020;59:524-33.

56 Ibañez D, Gladman DD, Touma Z, et al. Optimal frequency of visits for patients with systemic lupus erythematosus to measure disease activity over time. J Rheumatol 2011;38:60-3.

57 Zavodovsky BV, Sivordova LE. Cardiovascular safety of non-steroidal anti-inflammatory drugs in chronic inflammatory rheumatic diseases. Ter Arkh 2018;90:101-6.

58 Munguia-Realpozo P, Mendoza-Pinto C, Sierra Benito C, et al. Systemic lupus erythematosus and hypertension. Autoimmun Rev 2019;18:102371.
59 Chinthapalli K. High dose NSAIDs may double the risk of heart attacks and heart failure, says new study. BMJ 2013;346:f3533.

60 Marsico F, Paolillo S, Filardi PP. NSAIDS and cardiovascular risk. $J$ Cardiovasc Med 2017;18:e40-3.

61 Mukherjee D, Nissen SE, Topol EJ. Risk of cardiovascular events associated with selective COX-2 inhibitors. JAMA 2001;286:954-9.

62 Simon LS. Non-steroidal anti-inflammatory drugs and their benefits and harms: the challenge of interpreting meta-analyses and observational data sets when balanced data are not analyzed and reported. Arthritis Res Ther 2015;17:130. 